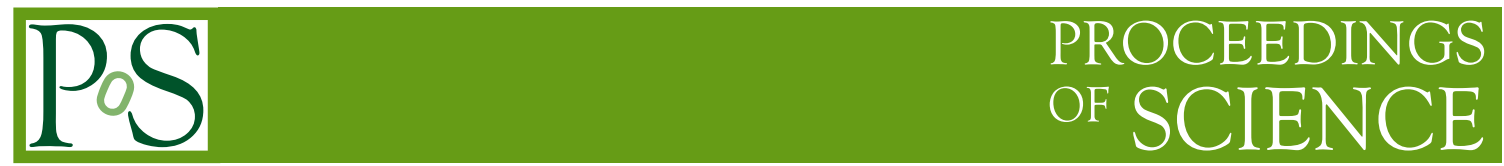

\title{
DC muon beam related physics and experiments
}

\section{Toshiyuki Iwamoto*}

ICEPP, The University of Tokyo, 7-3-1 Hongo, Bunkyo-ku, Tokyo 113-0033, Japan

E-mail: iwamoto@icepp.s.u-tokyo.ac.jp

The DC muon beam related experiments, mainly about charged lepton flavor violating muon decay search experiments like MEG/MEG II and Mu3e are described as well as their physics motivation and the accelerators. Prospects for the upgrade plan of DC muon beam and the experiments are also discussed.

The 19th International Workshop on Neutrinos from Accelerators-NUFACT2017

25-30 September, 2017

Uppsala University, Uppsala, Sweden

${ }^{*}$ Speaker. 


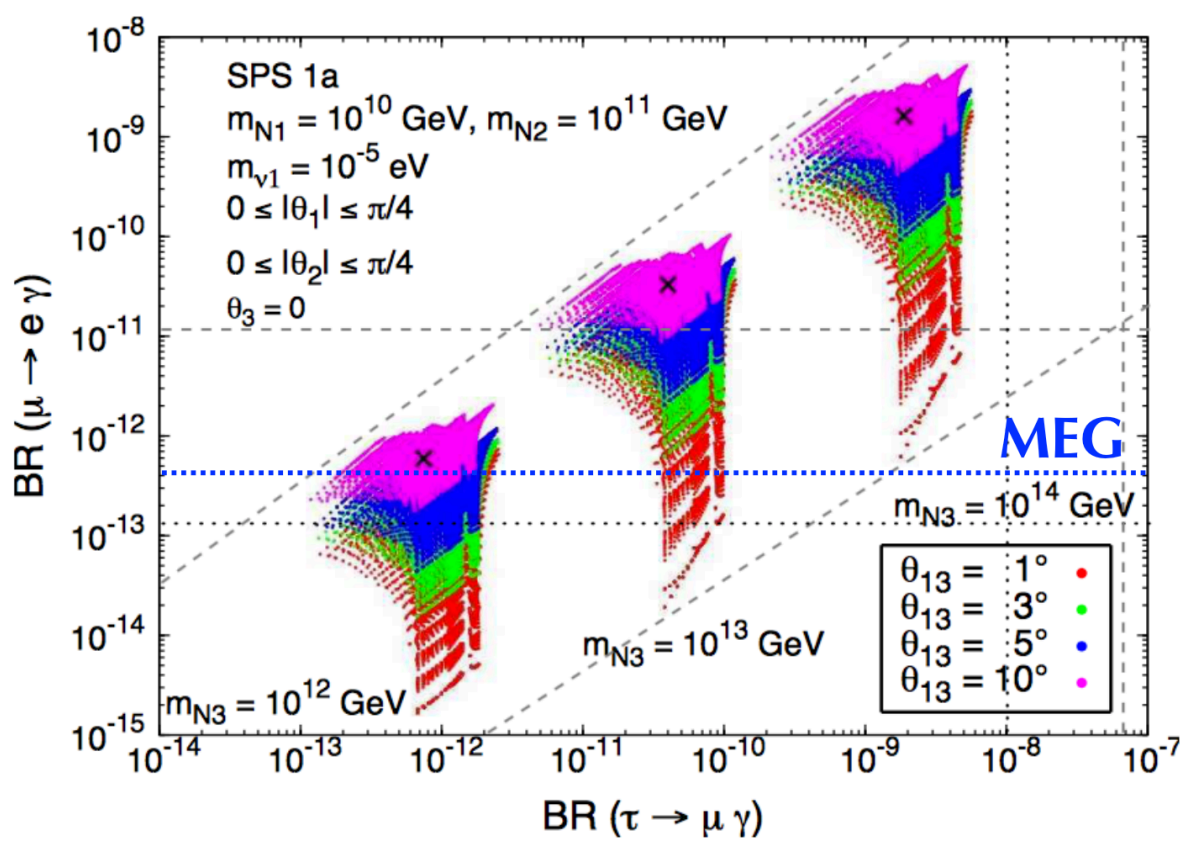

Figure 1: A prediction of a relation between $\mu \rightarrow e \gamma$ and $\tau \rightarrow \mu \gamma$ charged lepton flavor violating decays from a SUSY-Seesaw model[1]

\section{Introduction}

The standard model (SM) of the particle physics describes the electromagnetic, weak, and strong interactions as well as all known elementary particles, and can explain most experimental results. All the components for SM are already lined up after the higgs discovery in 2012. On the other hand, SM can not explain their many parameters contained in the model, dark matter, and gravity, and we consider this model is a low energy approximation of more fundamental theory. We are therefore looking for such new physics beyond the SM. One of the methods is to search for lepton flavor violation (LFV) phenomena. LFV is practically forbidden in the SM, but it is experimentally observed in neutrino oscillations. In charged lepton sector, LFV has not been observed yet. Prediction of the branching ratio of $\mu^{+} \rightarrow e^{+} \gamma$ taking into account neutrino masses and oscillations can be calculated to be $10^{-54}$, which is not measurable. This means that once we find any charged LFV phenomena, we find new physics beyond SM. Many new physics like supersymmetric grand unified theory (SUSY-GUT), SUSY-seesaw, extra dimension however predict large charged LFV rates. Figure 1 shows one example of new physics from a SUSY-seesaw model.

The $\mu \rightarrow e \gamma$ and $\tau \rightarrow \mu \gamma$ correlation is shown depending on heavy neutrino mass, $\theta_{13} . \theta_{13}$ value is measured to be $8-9^{\circ}$, which enhances the $\mu \rightarrow e \gamma$ branching ratio. Observation of Charged LFV is clear evidence for new physics beyond the SM, and Charged LFV experiments already reach the region expected by new physics. For example, the MEG experiment restricted the region above the blue line in Fig.1. 


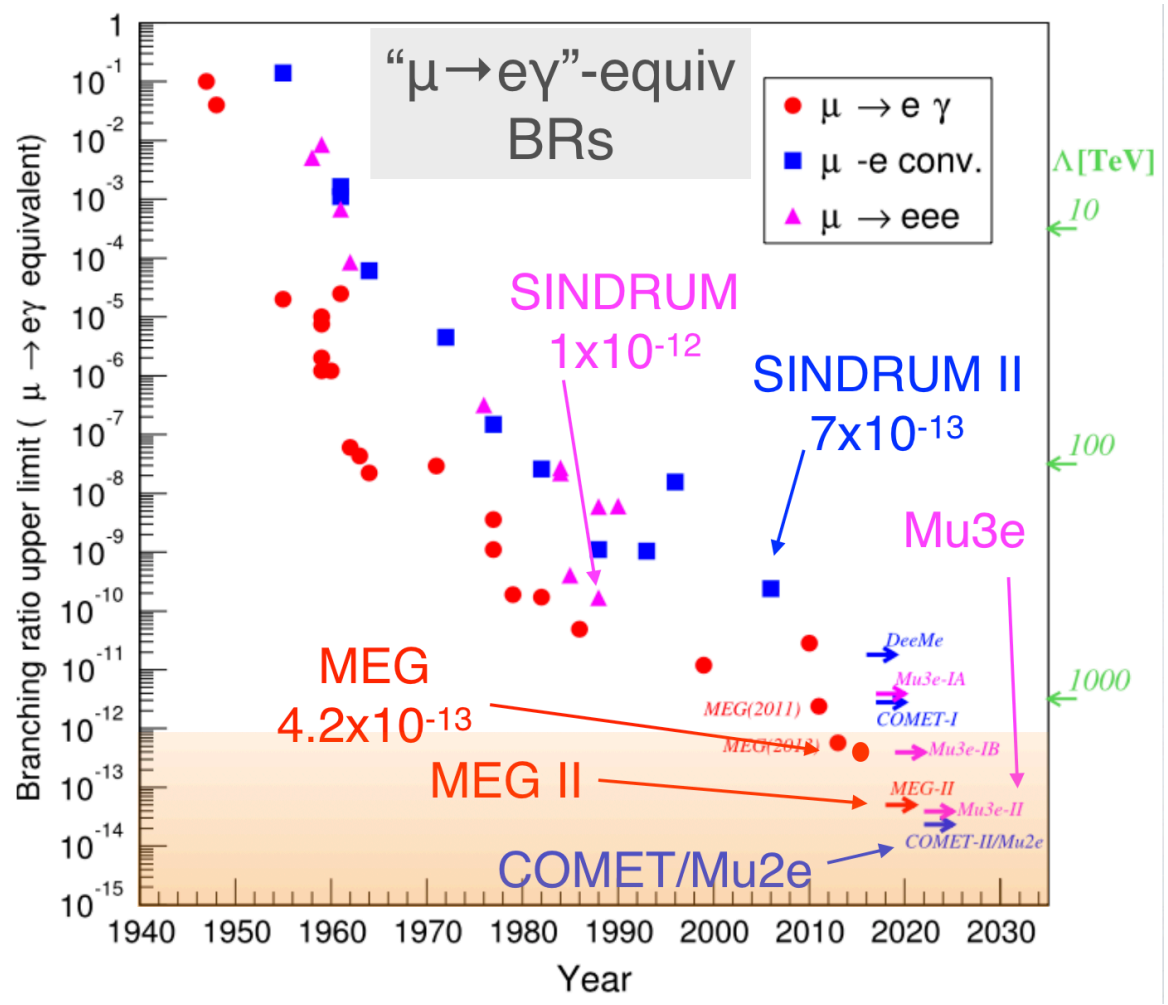

Figure 2: History of Charged LFV search. The vertical axis is the $\mu \rightarrow e \gamma$ equivalent branching ratios which assumes that the LFV is dominated by loop contribution ( or photonic process), additional coupling contribution is taken into account in the figure to compare these decay mode directly. Previous experiments, and proposed projects are also shown[3].

Muons are very clean, and can be a sensitive probes to study LFV. Currently intense muon beams are available, and that is why this mode is used intensivey to look for new physics. Charged LFV search has a long history as shown in Figure 2. The MEG experiment has a world strongest branching ratio upper limit for $\mu^{+} \rightarrow e^{+} \gamma$ decay mode, $4.2 \times 10^{-13}$ [2]. Many projects like MEG II, COMET/Mu2e, and Mu3e will start to search for different decay modes within a decade.

$\mu^{+} \rightarrow e^{+} \gamma$ process is sensitive to the loop (photonic) contribution, and $\mu$-e conversion or $\mu \rightarrow$ eee process is also sensitive to contact terms (non-photonic). In order to pin down the new physics, results of different branching ratios from these different decay modes will be important.

\section{DC muon beam}

Muons are produced from pion decays, and the pion production yield depends on the proton accelerator power. Paul Scherrer Institute (PSI) in Switzerland has a proton acclelerator with a $2.4 \mathrm{~mA}$ cyclotron at $590 \mathrm{MeV}$ which has the world strongest power, $1.4 \mathrm{MW}$. Many projects to have multi-MW potential are planned in the world for different purposes. Intense muon beams are available at PSI which makes a DC muon beam, and at J-PARC in Japan and Fermilab in US which make pulsed muon beams. The PSI accelerator produces the most intense DC muon beam larger than $10^{8} \mu^{+} / \mathrm{s}$, and produces the spallation neutrons as much as $10^{14} \mathrm{n} / \mathrm{s}$ at the same time. This 
accelerator has a possibility to be upgraded up to $3 \mathrm{~mA}$ if they replace RF cavities. One interesting thing is that the current best limits of Charged LFV are all from PSI, $\mu^{+} \rightarrow e^{+} \gamma<4.2 \times 10^{-13}$ by MEG in 2016, $\mu \rightarrow e<7 \times 10^{-13}$ by SINDRUM II in 2006[4], and $\mu \rightarrow e e e<1 \times 10^{-12}$ by SINDRUM in 1988[4].

\section{Experiments with DC muon beams}

\subsection{MEG/MEG II Experiment}

A DC beam is suitable for coincidence experiments like $\mu^{+} \rightarrow e^{+} \gamma$ or $\mu \rightarrow e e e$, and a pulsed beam is suitable for non-coincidence experiments like $\mu-e$ conversion. $\mu^{+} \rightarrow e^{+} \gamma$ signals are back-to-back events with timing coincident, energy of $\mathrm{e}^{+}$and $\gamma$ are $52.8 \mathrm{MeV} . \mu \rightarrow$ eee signals have a common vertex, coincident timing, the sum of momentum is zero, and the sum of energy is muon mass. There are two main background for this mode, accidental $e^{+}$and $\gamma$, and radiative muon decay $\mu \rightarrow e v v \gamma$. Accidental background is dominant for $\mu^{+} \rightarrow e^{+} \gamma$. The number of accidental backgrounds is proportional to the muon beam rate square, and each detector resolution, and it is important to keep the instantaneous beam rate low and the detector resolutions good. There are two backgrounds for $\mu \rightarrow e e e$, accidental eee and $\mu \rightarrow e e e v \nu$.

The MEG experiment performed data taking from 2008 to 2013 to search for lepton flavor violating $\mu \rightarrow e \gamma$ mode using $3 \times 10^{7} \mu / s$ of a beam intensity at PSI. Muons are stopped at a target, and positron trajectories are bent by superconducting solenoid (COBRA), and tracked by segmented drift chambers. Timing is measured with scintillation bar timing counter. $\gamma$ is measured with liquid xenon (LXe) detector with 846 PMTs. Final results were pulibshed in 2016. There was no signal candidate, and the branching ratio upper limit of $4.2 \times 10^{-13}$ is set. At that time, the sensitivity improvement was already limited by accidental background, and the experiment seriously considered detector upgrade. In 2013, a proposal[5] to upgrade the MEG detector and to achieve ten times better sensitivty as quick as possible, which is called MEG II experiment.

Figure 3 shows the MEG II concept. The muon beam rate will be doubled. Positron tracking will be done with cylindrical homogeneous drift chamber. Timing is measured with pixelated timing counter. $\gamma$ is measured with 4092 MPPCs for $\gamma$ incident face, and 668 PMTs for remaining faces. A new detector to detect radiative muon decay background is installed. Next year, we will start engineering run with all the detectors, and physics run will follow. We will reach $4 \times 10^{-14}$ sensitivity within three years, which is ten times improvement from MEG.

The MEG II positron tracker uses single volume wire drift chamber with 1200 sense wires with $1.93 \mathrm{~m}$ long, $20 \mu \mathrm{m}$ diameter. Hit resolution is improved, and high granularity and increased number of hits per track enables all the resolution improvement by a factor of 2 , momentum resolution from $310 \mathrm{keV}$ to $130 \mathrm{keV}, \theta$ angle resolution from 9.4 to $5.3 \mathrm{mrad}, \phi$ angle resolution from 8.7 to $3.7 \mathrm{mrad}$. Due to less material budget, and high transparency towards the timing counter is realized, and the detection efficiency is improved from 30 to $70 \%$.

Higher granularity scintillator, pixelated by $2 \times 256 \mathrm{BC} 422$ scintillator plates with the size of $120 \times 40$ (or 50$) \times 5 \mathrm{~mm}^{3}$ readout by SiPMs at both ends. Including multi-hit condition per track we can obtain better timing resolution like 70 ps to $30 \mathrm{ps}$.

In order to detect $52.8 \mathrm{MeV} \gamma, 900 \mathrm{~L}$ liquid xenon $\gamma$ calorimeter is used with 4092 new VUVsensitive SiPM which is developed in collaboration with Hamamatsu (MPPC) installed on the $\gamma$ 


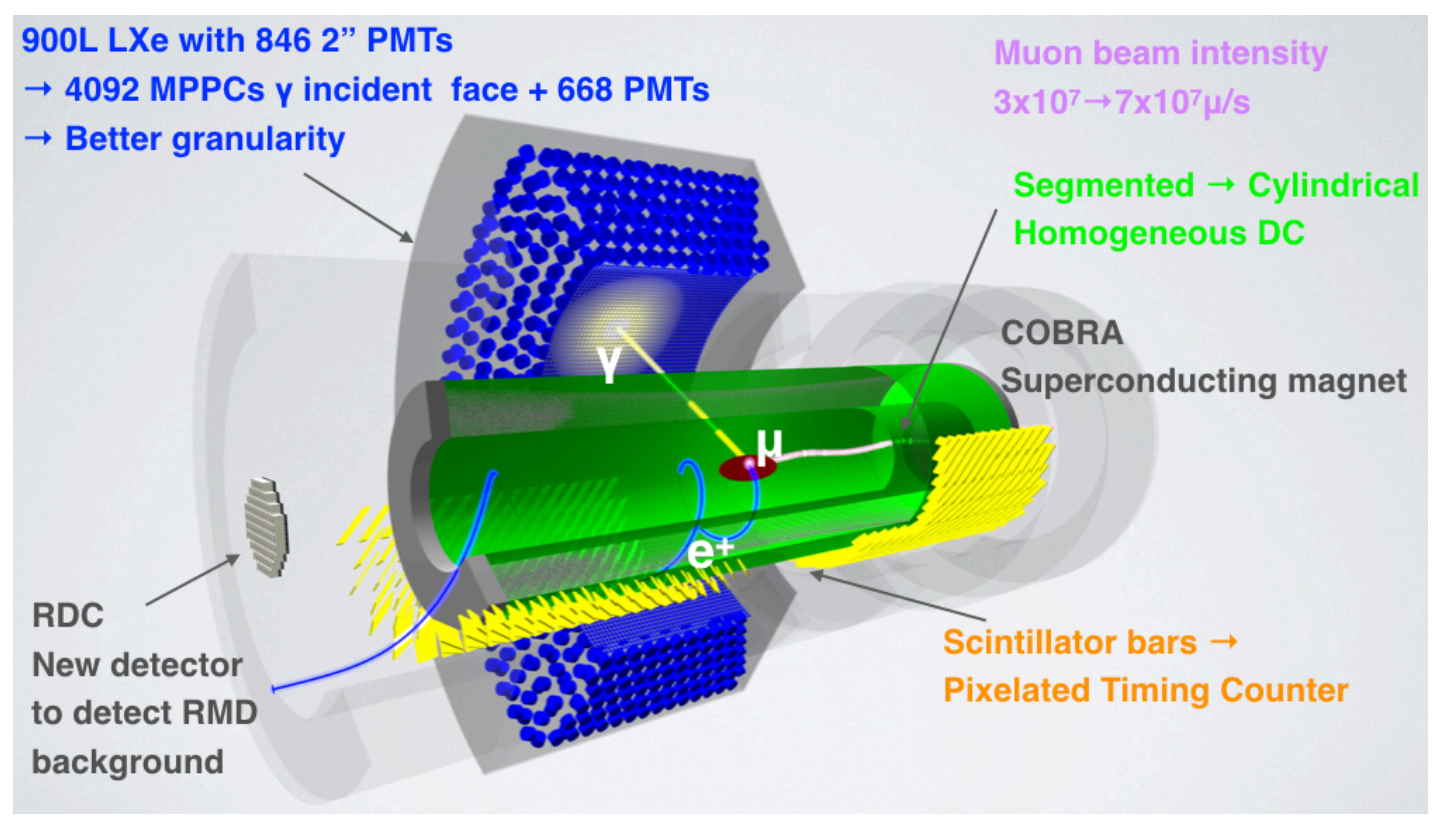

Figure 3: MEG II detector

incident face. Due to higher granularity for $\gamma$ incident face, we can expect twice better resolution for position and energy, and slight improvement for timing. Due to less material budget in front of $\gamma$ incident face of smaller photo sensors, the detection efficinecy is also improved from 65 to $70 \%$.

A new additional detector for the MEG II to identify $\mu \rightarrow e v v \gamma$ by tagging low momentum $\mathrm{e}^{+}$ associated with high energy $\gamma$. To detect low momentum positron, we use 12 plastic scintillators plus 76 LYSO crystals. The construction is finished, and expected to further reduce the background from radiative muon decay by $40 \%$.

Timing counter construction is finished, and ready for the beam time of this year. The LXe detector construction is finished, too, and purification and detector commissioning is in progress. The construction of drift chamber is in progress, and this will be delivered to PSI in early 2018. In 2018, the MEG II experiment will start the engineering run, and reach $4 \times 10^{-14}$ in three years.

\subsection{Mu3e Experiment}

The Mu3e experiment is a new search for the lepton-flavour violating decay of a positive muon into two positrons and one electron, and the proposal was presentd to PSI in 2013[6]. This experiment will use the PSI muon beam line in $\pi \mathrm{E} 5$ at the full available beam intensity from the beginning. Figure 4 shows the detector concept. Electron/positron trajectories are measured with very thin tracking detector, Mupix, with $50 \mu \mathrm{m}$ thickness. Timing is measured with fiber hodoscope at the inner tracker with $500 \mathrm{ps}$ resolution. Outer region of tile detector will measure the timing with 70 ps resolution. The central tracker has four layers and re-curl tracker has two layers. Because 


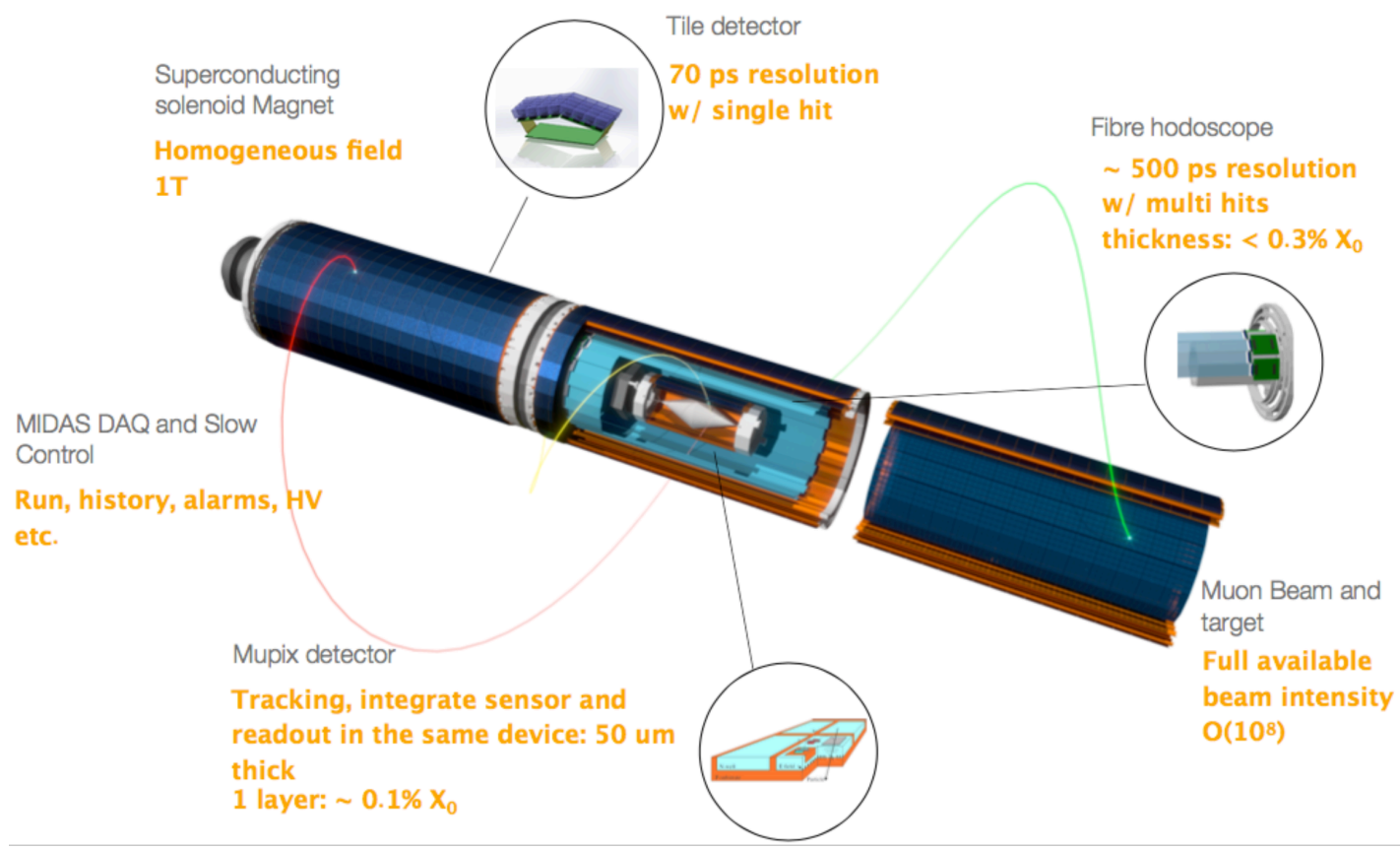

Figure 4: Mu3e detector

multiple scattering dominates the momentum resolution, minimum material budget is necessary to achirve $0.5 \mathrm{MeV} / \mathrm{c}$ momentum resolution.

The high voltage monolithic active pixel sensor, called HV-MAPS is used for the tracker, and this has a ultra-light weight mechanics with $50 \mu \mathrm{m}$ silicon sensor, $25 \mu \mathrm{m}$ capton flex print with $\mathrm{Al}$ traces, and $25 \mu \mathrm{m}$ capton support frame. The time resolution is not very good like $20 \mathrm{~ns}$, and it requires timing detectors. Active area of each chip is $20 \times 20 \mathrm{~mm}^{2}$, and these are under development.

To detect timing of those electron and positrons, they prepare two different counters. For the central region before outer pixel layers, square or round shaped $250 \mu \mathrm{m}$ scintillating fibers are used with SiPM-based readout. Three layers are prepared with $32 \mathrm{~mm}$ wide, $290 \mathrm{~mm}$ long fibers. The timing resolution will be $1 \mathrm{~ns}$. Outer tracker region after recurl pixel layers has scintillation tiles with the size of $6.5 \times 6.5 \times 5.0 \mathrm{~mm}^{3}$ readout by SiPMs, which has $100 \mathrm{ps}$ resolution.

The delivery of the magnet is delayed because the original plan was cancelled. The updated plan of the delivery is in 2019. Pixel tracker prototype test is in progress, and currently Mupix 8 first full size of sensor $\left(1 \times 2 \mathrm{~cm}^{2}\right)$ has arrived in August 2017, and this is intensively tested. Scintillating fiber's prototype of round(Kuraray SCSF-81M) and squaread(Saint-Gobain BC418) shapes are tested, and $600 \mathrm{ps}$ timing resolution is already achieved. For scintillating tile detector, the promising results of the timing resolution from $4 \times 4$ array is also obtained to be around 70 ps. Engineering run is expected in 2019 to reach the sensitivity of $2 \times 10^{-15}$ in Mu3e phase I with $10^{8} \mu / s$. The $\pi \mathrm{E} 5$ beamline at PSI is shared by MEG II and Mu3e, and to allow the smooth beam time sharing, MEG II detector can be there even in Mu3e beam time thanks to the compact muon beam line for Mu3e 
which only requires the upstream side space.

\section{Prospects of DC muon beam and the charged LFV experiments}

HiMB (High Intensity Muon Beamline) project is now discussed at PSI, which is an upgrade plan of the high intensity muon beam line. Existing target (Target M) will be replaced with new $20 \mathrm{~mm}$ effective length with $5^{\circ}$ slanted target, and $1.3 \times 10^{11} \mu$ can be generated. If a capture solenoid of $0.35 \mathrm{~T}$ is put at the distance of $250 \mathrm{~mm}, 3.4 \times 10^{10} \mu / \mathrm{s}$ can be trapped, and after the solenoidal beam line, $1.3 \times 10^{10} \mu / \mathrm{s}$ can be transmitted to experimental sites. This is promising, and the next plan is the feasibility study of slant target at target $\mathrm{E}$.

Mu3e phase II is planned with an increased muon stopping rate to $2 \times 10^{9} \mu / \mathrm{s}$, and additional recur stations will increase acceptance for recurler to reach $10^{-16}$ level.

If the $\gamma$ resolutions expected at MEG II are achieved, further improvements from the detector will be difficult, and other $\gamma$ detection methods must be developed. In general, calorimeter methods can get high efficiency and good resolution, but higher light yield and fast response are required. On the other hand, photon conversion methods have typically low efficiency but extreme high resolution and phtoon direction. In order to get high efficiency as well as good energy and angle resolutions, the optimization of the converter thickness is necessary. If we put sensitivity as a function of the muon beam rate, photon conversion method is better in the higher beam rate, and calorimeter is still good in the midium rate. Some examples of photon conversion approach or caloriemter approach show that we may be able to reach a few $10^{-15}$ level within three years running, and more precise study must be performed[7].

\section{Muon precision physics}

A promising method for muon cooling[8] is studied at PSI in detail. The main physics targets of the development of the muon cooling are mainly muonium spectroscopy, gravity of antimatter, $\mu \mathrm{SR}$, muon g-2, or muon EDM. By making temperature gradient which produces density gradient in the target, transverse compression can be done in electric and magnetic fields. For the longitudinal compression we can use electric field at room temperature. Both compression methods are already demonstrated independently. Next step is to combine both two stages into one.

\section{Summary}

Charged LFV experiments with DC muon beam have real chance to find new physics. MEG II / Mu3e / DeeMe / COMET / Mu2e will start within 5 years. Upgrade projects of DC muon beam are ongoing, and next generation experiments should follow with new technologies.

\section{References}

[1] S. Antusch, E. Arganda, M. J. Herrero and A. M. Teixeira, JHEP 0611, 090 (2006) doi:10.1088/1126-6708/2006/11/090 [hep-ph/0607263].

[2] A. M. Baldini et al. [MEG Collaboration], Eur. Phys. J. C 76, no. 8, 434 (2016) doi:10.1140/epjc/s10052-016-4271-x [arXiv:1605.05081 [hep-ex]]. 
[3] T. Mori and W. Ootani, Prog. Part. Nucl. Phys. 79, 57 (2014). doi:10.1016/j.ppnp.2014.09.001

[4] C. Patrignani et al. [Particle Data Group], Chin. Phys. C 40, no. 10, 100001 (2016). doi:10.1088/1674-1137/40/10/100001

[5] A. M. Baldini et al., arXiv:1301.7225 [physics.ins-det].

[6] A. Blondel et al., arXiv:1301.6113 [physics.ins-det].

[7] G. Cavoto, A. Papa, F. Renga, E. Ripiccini and C. Voena, arXiv:1707.01805 [hep-ex].

[8] D. Taqqu, Phys. Rev. Lett. 97, 194801 (2006). doi:10.1103/PhysRevLett.97.194801 